\title{
Eine neue Kartographie des Möglichen
}

\author{
Rezension zu Nikolai Roskamm (2017): Die unbesetzte Stadt. Postfundamentalis-
} tisches Denken und das urbanistische Feld. Basel/Berlin: Birkhäuser Verlag.

Erol Yildiz

Abb. 1 Titel des Buches (Quelle: Birkhäuser Verlag)

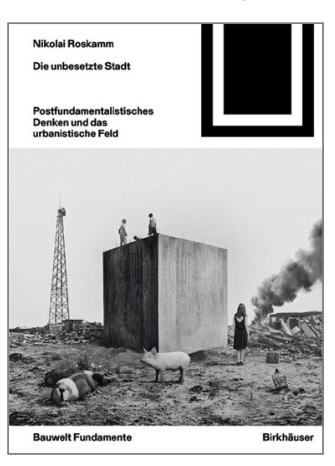

Es gibt unterschiedliche Perspektiven, aus denen Stadt beobachtet, beschrieben und analysiert werden kann. Abhängig vom jeweiligen Standpunkt gerät dabei, wie in einem Episodenfilm, ein anderes Phänomen, eine andere Relevanzstruktur und damit eine andere Version der Wirklichkeit ins Blickfeld. Und so scheint trotz immer neuer Theorien von der Stadtgesellschaft nicht viel mehr übrig als ein „unheilbar plurales Konzept“, wie Armin Nassehi (1998: 158) einmal festgestellt hat.

Der postfundamentalistische Ansatz, der in dem Buch von Nikolai Roskamm entfaltet wird, ist ein weiterer Blickwinkel auf die Stadt: als ein hybrides und unvollständiges Konstrukt. Der Titel des vorliegenden Bandes Die unbesetzte Stadt signalisiert eine interessante Idee, die aus unterschiedlichen Theorieperspektiven und in unterschiedlichen Kontexten beleuchtet wird. Der Untertitel Postfundamentalistisches Denken und das urbanistische Feld benennt dabei eine spezifische Denkhaltung, die die theoretische Abhandlung durchzieht. Schlüsselbegriffe wie Antagonismus, Konflikt, Kontingenz, unstabil, unbesetzt, unvollständig et cetera erscheinen für diesen Ansatz konstitutiv.

Einleitend wird die Idee des Postfundamentalismus in ihrem Kern und ihrer Abgrenzung zu anderen Konzepten vorgestellt. Um seine Theorie zu begründen, bezieht sich der Autor in erster Linie auf zwei Werke von Oliver Marchart, in denen dieser seine postfundamentalistische Gesellschaftstheorie entwickelt hat: Die politische Differenz (2010) und Das unmögliche Objekt der Gesellschaft (2013). Marchart kann aus dieser Sicht als ein expliziter ,Postfundamentalist' bezeichnet werden. Der Grundgedanke von Roskamm ist nun, die von Marchart entfaltete postfundamentalistische Gesellschaftstheorie auf das urbanistische Feld anzuwenden. Darüber hinaus begibt er sich auf die Suche nach postfundamentalistischen Ansätzen in der politischen Philosophie etwa bei Karl Marx, Claude Lefort, Ernesto Laclau, Michel Foucault oder Jacques Derrida. Insbesondere Henri Lefebvre, der sich in seinen Schriften explizit mit Stadt und Urbanität auseinandergesetzt hat, spielt für Roskamms Theoriekonstruktion zur unbesetzten Stadt eine richtungsweisende Rolle. Es werden hier also einzelne theoretische Aspekte 
aufgegriffen, als postfundamentalistisch gelesen und dieser Denkrichtung zugeordnet. Das kritische Grundanliegen besteht darin, „den Stadtbegriff von der sozialwissenschaftlichen auf eine sozialtheoretische Ebene zu verschieben, von der Ebene des Sozialen, Empirischen und Partikularen auf die Ebene des Politischen, Theoretischen und Totalen “(9). Mit anderen Worten: „Wirklich besteht das Ziel einer sozialtheoretischen Kritik darin, den sozialwissenschaftlichen Regelbetrieb zu hinterfragen und herauszufordern.“(12)

Den Kern des so genannten postfundamentalistischen Denkens macht die Auffassung aus, dass es keine Letztbegründungen und keine Fundamente, auf denen alles andere aufgebaut werde, geben könne. Historische Prozesse seien ohne kontingente und konflikthafte Kräfte nicht denkbar. „Kontingent bedeutet, dass alle sozialen Dinge und Abläufe grundsätzlich auch anders sein können, dass nichts aus sich heraus und von vornherein (vor)bestimmt ist. Eine solche Kontingenzbehauptung reproduziert wiederum die These vom Nicht-Vorhandensein von Letztbegründungen." (13) Der Stadtbegriff wird von Roskamm negativ bestimmt, als ein instabiler Ort, der niemals komplett besetzt werden könne. Dieser Gedanke lehnt sich an Claude Lefort an, für den der Ort der Macht in der Demokratie unbesetzt bleibt (vgl. 22). Die Idee vom leeren Ort der Macht, die für Lefort eine wesentliche Voraussetzung für das Politische darstellt, spielt für die Grundannahmen des postfundamentalistischen Denkens eine zentrale Rolle. „Leforts Konzept vom leeren Ort der Macht ist damit äußerst geeignet, einer postfundamentalistischen Theorie der Stadt den Weg zu bahnen“, so Roskamm (25).

Im ersten Teil werden zunächst Theorien und Fundamentalismen kritisiert, die im akademischen Kontext endgültige Wahrheiten produzieren, und die unter den Oberbegriff des Determinismus zusammengefasst werden können - eine Denkrichtung, die kaum andere Möglichkeiten zulässt (vgl. 28). Aus postfundamentalistischer Sicht wird Stadt als Ort des Kampfes und der Auseinandersetzung definiert. Dabei bezieht sich der Autor auf Karl Marx und Friedrich Engels, nach denen es aus historischer Sicht keine festen und stabilen Fundamente gebe, sondern allenfalls „prekäre Streitsachen“ (84). In diesem Kontext verweist der Autor darauf, dass im Marx'schen Historischen Materialismus im Allgemeinen die Grundlagen für kritisches Denken angelegt seien.

Nachdem die Bedeutung des Historischen Materialismus für eine kritische Stadtforschung diskutiert wurde, setzt sich Roskamm mit Lefebvres Überlegungen zu Stadt und Urbanität auseinander. Er versucht, wesentliche Grundgedanken daraus für eine postfundamentalistische Stadttheorie nutzbar zu machen. In Lefebvres theoretischen Ausführungen erkennt er zu Recht eine Denkhaltung, die den Marxismus in eine postfundamentalistische Richtung bewegt hat, denn die Theoriekonstruktion Lefebvres lässt keine Letztbegründung zu (vgl. 101).

In diesem Zusammenhang erscheint mir die Idee vom ,Recht auf Stadt', die mit Lefebvres Überlegungen begründet werden kann, aktuell und zukunftsweisend. Auch die Unterscheidung zwischen urbaner Praxis (tatsächlicher Lebenspraxis der Menschen in der Stadt) und urbanistischer Praxis (offizieller Stadtplanung und Stadtpolitik) ist im Hinblick auf aktuelle Debatten von großer Relevanz (vgl. 128f.). Zwei Hauptaspekte, die Lefebvres Stadttheorie ausmachen und die von Roskamm für ein postfundamentalistisches Denken 
hervorgehoben werden, sind hier zu nennen. Einerseits wird Stadt als Ort des Konflikts gedacht, andererseits als ein Ort des Möglichen konzipiert. Stadt eröffnet Menschen Räume zum Experimentieren, ,Zwischenräume‘, in denen widerständige Praktiken entfaltet werden können. Dabei gehe es bei Lefebvre nicht um eindeutige Räume, sondern um Brachen, Nischen oder Lücken, die die eigentliche Substanz der Stadt ausmachen (vgl. 144). Anschließend werden zwei ähnliche Konzepte im Hinblick auf ihre Relevanz für postfundamentalistisches Denken hervorgehoben: Antagonismus und Kontingenz. Roskamm bezieht sich dabei vor allem auf Laclau, für den ein Signifikant notwendigerweise unbesetzt bleibt:

„Das, was Laclaus Begriff des leeren Signifikanten bezeichnet, gleicht nicht zufällig der Überschrift, die ich für mein hier insgesamt ausgebreitetes Vorhaben gewählt habe. Laclaus Theorie offenbart eine weitere, wenn nicht gar die eigentliche Bedeutung der These von der unbesetzten Stadt. Mit Laclau gesprochen bedeutet das, dass Stadt unbesetzt ist, weil sie ein Signifikant ist. Die These von der unbesetzten Stadt bestimmt Stadt zunächst als leeren und kontingenten Signifikanten.“(191).

Kontingenz wird von Roskamm als zweites ,postfundamentalistisches Fundament $t^{6}$ bestimmt. Er spricht hier von einer Notwendigkeit der Kontingenz (vgl. 212). Dabei bezieht er sich auf Hannah Arendts Begriff des öffentlichen Raums, der als ein Zwischenraum definiert wird. Öffentlicher Raum wird zu einem unbesetzten Zwischenraum, zu einem leeren, kontingenten Raum (vgl. 217). In den Worten Roskamms: „Für eine poststrukturalistische Stadttheorie ist es ein vielversprechender Perspektivwechsel, Stadt kontingent zu setzen und daher nicht mehr danach zu fragen, was Stadt ist, sondern was sie verunmöglicht - also zu ergründen, was verhindert, dass Stadt mit sich identisch wird, was unterbindet, dass sie jemals einen komplett erschlossenen und besetzten Aggregatzustand erreicht.“ (230) Auch die Genealogie Foucaults wird aus diesem Blickwinkel betrachtet, insofern seine Methode den gewohnten Essentialismus kritisch hinterfragt, dessen metaphysische Fundamente radikal in Frage stellt und auf eine kontingente Positionierung verweist (vgl. 246).

Stadt sozialtheoretisch zu denken, bedeutet für den Autor der vorliegenden Abhandlung nicht, eine Art neue Philosophie der Stadt zu erfinden. Es gehe ihm vor allem darum, aus seinen Ideen zum Postfundamentalismus eine kritische Stadtforschung zu begründen, die sich mit dem herkömmlichen Urbanismus kritisch auseinandersetzt und daraus eine „Praxis der Kritik“ entwirft (279). „Die auf dem urbanistischen Feld verortete kritische Stadtforschung scheut es, die eigene Grundierung zu dekonstruieren“, so Roskamm (280).

So erweist es sich als Grundannahme dieser postfundamentalistischen Perspektive, dass Stadt nicht durch das Anwesende, sondern durch das Abwesende zu bestimmen ist und dadurch unbesetzt ist beziehungsweise bleibt. Dies wird im letzten Kapitel noch einmal unterstrichen (vgl. 350). In diesem Kontext taucht der Begriff der Irritation auf, der sich aus der Bedeutung der unbesetzten Stadt als „unmögliches Objekt“ ergibt, das „nicht messbar und der traditionellen wissenschaftlichen Analyse nur begrenzt zugänglich ist, 
weil es dem sozialwissenschaftlichen Zugriff immer wieder entgleitet und nachhaltige Verstimmungen hervorruft“ (388).

Abschließend wird nochmals bekräftigt, welche Bedeutung ein postfundamentalistischer Ansatz im urbanistischen Feld entfalten und welche Perspektiven auf Stadt sich aus dieser Interpretation des Politischen eröffnen kann. „Die unbesetzte Stadt eröffnet einen Raum, in dem es möglich ist, zu agieren, zu handeln, Dinge zu tun und zu gebrauchen“, so Roskamm (383). Weiter heißt es:

„Das postfundamentalistische Kontingenzpostulat ist Voraussetzung dafür, die Welt - und das heißt vor allem: die Welt der Ungerechtigkeit, Ungleichheit und Unterdrückung - nicht unveränderbar sein zu lassen. Seine Welterklärung findet der Postfundamentalismus im Antagonismus als Grundeigenschaft des Sozialen und Urbanen, und damit stellt er sich in eine postmarxistische Traditionslinie.“ (388)

Ein wesentliches Verdienst des vorliegenden Buches ist es, klassische Texte mit einem veränderten Fokus neu zu lesen, unterschiedliche Elemente zu einer postfundamentalistischen Denkhaltung zu verdichten, die, bezogen auf das urbanistische Feld, essentialistische und dualistische Konzeptionen radikal in Frage stellt, das bisher Ungedachte, Übersehene und Marginalisierte zum Ausganspunkt nimmt und neue, alternative Perspektiven auf Stadt und Urbanität eröffnet. Hier stellt sich allerdings die Frage, ob die konventionelle Stadtentwicklung beziehungsweise Stadtpolitik eine solche postfundamentalistische Perspektive überhaupt zur Kenntnis nehmen und in zukünftige Konzeptionen übersetzen wird. Oder driften vielmehr die von Lefebvre formulierten diametral entgegengesetzten Praxisformen, nämlich urbane (alltägliche) und urbanistische Praktiken (offizielle Stadtplanung) weiter auseinander? Die Idee der ,unvollständigen Stadt‘ von Richard Sennett (2018), die er in seinem aktuellen Buch beschreibt, wäre hier ebenfalls weiterführend (Sennett spricht sich auch für ein „unvollständiges Bauen“ aus).

Aus gutem Grund plädiert Roskamm dafür, Phänomene, die bisher getrennt betrachtet wurden, miteinander in Bezug zu setzen, Stadtforschung und Postfundamentalismus zusammenzudenken (vgl. 30). Auch die Idee Lefebvres, den Dualismus zwischen dem Ding Stadt und der Idee Stadt zu überwinden und beides zusammenzudenken, weist in diese Richtung. Aus philosophischer Sicht also eine „non-dualistische“ Perspektive abseits polarisierender Deutungen (vgl. Mitterer 2011). Hier wäre anzumerken, dass statt der Etablierung eines neuen Leitbegriffs wie ,Postfundamentalismus durchaus unterschiedliche Ansätze postmoderner, konstruktivistischer und kritisch-nondualistischer Ansätze in der kultur- und sozialwissenschaftlichen Stadtforschung ein ähnliches Anliegen haben und zum gleichen Ziel führen können.

\section{Autor_innen}

Erol Yildiz ist Soziologe an der Universität Innsbruck am Institut für Erziehungswissenschaften und forscht zu interkulturellen und migrationssoziologischen Fragestellungen. erol.yildiz@uibk.ac.at 


\section{Literatur}

Marchart, Oliver (2010): Die politische Differenz. Zum Denken des Politischen bei Nancy, Lefort, Badiou, Laclau und Agamben. Frankfurt am Main: Suhrkamp.

Marchart, Oliver (2013): Das unmögliche Objekt. Eine postfundamentalistische Theorie der Gesellschaft. Frankfurt am Main: Suhrkamp.

Mitterer, Josef (2011): Das Jenseits der Philosophie. Wider das dualistische Erkenntnisprinzip. Weilerswist: Velbrück.

Nassehi, Armin (1998): Die „Welt“-Fremdheit der Globalisierungsdebatte. In: Soziale Welt 2/1998, 151-167.

Roskamm, Nikolai (2017): Die unbesetzte Stadt. Postfundamentalistisches Denken und das urbanistische Feld. Basel/Berlin: Birkhäuser Verlag.

Sennett, Richard (2018): Die offene Stadt. Eine Ethik des Bauens und Bewohnens. Berlin: Hansa Verlag. 